Cahiers $d u$ MONDE RUSSE

\section{Cahiers du monde russe}

Russie - Empire russe - Union soviétique et États indépendants

$51 / 4 \mid 2010$

Sciences humaines et sociales en Russie à l'Âge d'argent

\title{
Kenneth Pinnow, Lost to the Collective
}

\section{Grégory Dufaud}

\section{(2) OpenEdition}

Journals

Édition électronique

URL : https://journals.openedition.org/monderusse/7383

DOI : 10.4000/monderusse.7383

ISSN : $1777-5388$

Éditeur

Éditions de l'EHESS

Édition imprimée

Date de publication : 25 novembre 2010

Pagination : 704-706

ISBN : 978-2-7132-2316-7

ISSN : $1252-6576$

Référence électronique

Grégory Dufaud, «Kenneth Pinnow, Lost to the Collective », Cahiers du monde russe [En ligne], 51/4 I 2010, mis en ligne le 09 décembre 2011, consulté le 03 septembre 2022. URL : http://

journals.openedition.org/monderusse/7383; DOI : https://doi.org/10.4000/monderusse.7383

Ce document a été généré automatiquement le 3 septembre 2022.

Tous droits réservés 


\title{
Kenneth Pinnow, Lost to the Collective
}

\author{
Grégory Dufaud
}

\section{RÉFÉRENCE}

Kenneth PINNOW, Lost to the Collective. Suicide and the Promise of Soviet Socialism, 1921-1929. Ithaca - Londres : Cornell University Press, 2010, 276 p.

1 Afin de dissiper toute ambiguïté, une remarque liminaire : le livre de Kenneth Pinnow est une histoire non pas du suicide pendant la période de la NEP, mais de la manière dont les responsables et experts soviétiques lui donnèrent du sens. Décriminalisé en 1917, le suicide n'en resta pas moins un geste transgressif pour les bolcheviks qui s'interrogèrent à son propos. L'ouvrage est constitué de cinq chapitres qui donnent à voir différents points de vue sur le suicide. Pour ce faire, l'auteur s'appuie sur un matériau varié et solide : les documents issus des Archives centrales et régionales russes (Moscou, SaintPétersbourg, Tula et Jaroslavl') et des Archives centrales ukrainiennes ont été complétés par un corpus non moins important d'imprimés.

Cette enquête sur le suicide à l'époque soviétique prolonge chronologiquement les travaux d'Irina Paperno et de Susan Morrissey sur la Russie impériale ${ }^{1}$. L'auteur entend mettre au jour les techniques de gouvernement des hommes et les modalités de production du savoir que celles-ci requirent. On est là dans une perspective nettement marquée par le concept de "biopolitique» de Foucault (peut-être celui qui jouit aujourd'hui de la plus grande postérité; mais, curieusement, les travaux de Foucault n'apparaissent ici le plus souvent qu'à travers les commentaires qui ont pu en être faits). L'étude de Kenneth Pinnow est portée par une interrogation sur la construction du social et le rapport de l'individu à la société : comment les bolcheviks s'attelèrent-ils à refondre la société tsariste? Quel fut le rôle dévolu aux sciences sociales? Quelle était la place accordée à l'individu dans une société pensée comme collectiviste ? Ces questions sont au cœur de l'historiographie qui interroge la « modernité » de l'Union soviétique. 
Si elles ont la plupart du temps été traitées séparément, Kenneth Pinnow les noue ici ensemble et offre ainsi un propos d'une grande richesse.

3 Le premier chapitre fait l'archéologie du suicide comme problème social. Ce qui fut identifié comme une « épidémie de suicides » entre 1906 et 1914 permit à la communauté scientifique d'affirmer qu'il y avait là non seulement un problème moral, mais un enjeu de santé publique : les causes en furent discutées et des institutions créées pour recueillir des données empiriques. Médecins, statisticiens et autres intellectuels qui se voyaient en experts du social réclamaient un rôle que l'autocratie refusait de leur reconnaître. Ceux qui se rallièrent au régime bolchevik accédèrent aux postes qu'ils avaient longtemps espérés. La continuité du personnel fut aussi celle des idées et des concepts. Durant les années 1920, les débats sur le suicide furent relancés en raison de la nouvelle vague de morts volontaires concomitante au lancement de la NEP.

Parce que le suicide de communistes contredisait la conception selon laquelle le collectif était le lieu où se réalisait l'individu, les discussions à son sujet dans le parti furent nourries. Le deuxième chapitre s'intéresse aux réponses apportées par les responsables. Le suicide fut condamné en tant qu'il trahissait la cause socialiste et qu'il était un acte antisocial. Par son geste, le suicidé montrait qu'il n'était pas un authentique bolchevik. La hiérarchie du parti estima alors qu'un renforcement de l'esprit de groupe était à même de prévenir les suicides et, au-delà, de contenir les influences néfastes qui avaient pu les encourager. Elle pensait qu'un milieu sain devait permettre de développer conscience politique et esprit collectiviste. À cette fin, des efforts furent faits pour promouvoir davantage de camaraderie et renforcer la surveillance mutuelle au sein du " collectif ».

Le troisième chapitre s'intéresse à l'analyse du suicide par les experts de l'Institut médico-légal qui fut créé en 1919 par le Commissariat à la santé et dont le responsable était Jakov Lejbovič. Les experts légaux voyaient leur discipline comme une branche de la « médecine sociale » soviétique et estimaient, à ce titre, devoir jouer un rôle de tout premier plan dans le remodelage de la société. Leur étude du suicide chemina selon deux voies complémentaires. Elle procéda, d'une part, de la collecte de données quantitatives à l'échelle de toute la Russie. L'agrégat statistique fit émerger un corps social que les experts légaux pouvaient autopsier et ausculter tout comme ils le faisaient avec le cadavre des suicidés. C'est que ceux-ci s'employaient, d'autre part, à découvrir les particularités anatomiques en recourant à une approche anthropologique. Les recherches de la médecine légale étaient donc prises dans une tension entre le général et le particulier, le social et le biologique, qui renvoyait à la question de la nature humaine et à la possibilité de la transformer.

6 La construction d'un savoir sur le monde social à travers le suicide est examinée dans le quatrième chapitre qui porte sur le département de la statistique morale de la Commission centrale statistique. L'existence de ce dernier ne dépassa pas les années 1920, puisqu'il fut fermé en 1931. Pendant cette période, jouissant d'une grande autonomie, le département de la statistique morale s'employa à recueillir des données et à les interpréter à l'aune de divers facteurs afin de faire ressortir les spécificités des phénomènes sociaux. Dans la mesure où, depuis les travaux de Durkheim, il était accepté que le suicide relevât de déterminants socio-économiques, celui-ci pouvait représenter un étalon pour mesurer le chemin parcouru depuis la chute de l'autocratie. Or une étude sur le suicide parue en 1927 montrait le retard généralisé de la Russie sur l'Europe, infirmant ainsi le discours téléologique sur l'avènement prochain du socialisme. 
L'incapacité de la statistique morale à participer à ce récit fut probablement ce qui la condamna.

7 Le dernier chapitre de l'ouvrage porte sur l'Armée rouge, cet instrument de la révolution pensé comme un laboratoire du socialisme en train de se faire. L'Administration politique, qui y étudiait les morts volontaires, avançait que leurs recherches constituaient un outil à même de mesurer l'état moral et idéologique des soldats. Les catégories mobilisées étaient les mêmes que celles de la statistique morale, des médecins légaux et des sociologues européens. Mais elles étaient ici mises au service d'un idéal panoptique selon lequel tout un chacun devait être soumis au regard de l'autre et de la hiérarchie. D'après Kenneth Pinnow, la solution envisagée pour prévenir les suicides fut " a medicopolitics that promoted the comprehensive care of the individual as an integral component of the collective's existence and well-being»(p. 228). Par là, il s'agissait de créer la société harmonieuse imaginée par les bolcheviks.

8 Au terme de ce parcours kaléidoscopique, le suicide apparaît donc comme un révélateur des difficultés de l'État issu d'Octobre à se saisir du corps et de l'âme de ses sujets. Les moyens déployés pour le comprendre furent autant d'outils destinés à donner accès à la personnalité dans l'espoir de la remodeler. Pour Kenneth Pinnow, le suicide conduisit à l'individuation du social et confronta les bolcheviks à l'« agentivité » (agency) des individus. En mobilisant à plusieurs reprises ce concept aujourd'hui d'un usage fréquent dans les sciences sociales, l'auteur veut renvoyer à la capacité d'agir des agents. L'utilisation qu'il en fait est peut-être un peu floue, en ce sens qu'on ne voit pas toujours s'il entend par là la possibilité d'agir de manière autonome, les actions elles-mêmes, la réflexivité des agents sur leurs actions ou tout cela en même temps. Mais ce n'est là qu'un détail au regard de l'intérêt de la recherche réalisée par Kenneth Pinnow.

\section{NOTES}

1. Irina Paperno, Suicide as a Cultural Institution in Dostoevsky's Russia, Ithaca- Londres : Cornell University Press, 1997 ; Susan K. Morrissey, Suicide and the Body Politics in Imperial Russia, Cambridge, MA - Londres : Cambridge University Press, 2007. 